\title{
Función paterna y la subjetividad de los adolescentes en las Unidades Educativas Fiscales de Manta
}

\author{
Paternal function and the subjectivity of adolescents in the Manta Fiscal Educational Units
}

\begin{abstract}
Autoras
Amira Gioconda Trujillo Eljuri. Universidad Laica Eloy Alfaro de Manabi. Manta. Manabi. Ecuador. amiratrujillo@yahoo.com orcid.org/0000-0003-1856-1431

Inés Vera Molina. Universidad Laica Eloy Alfaro de Manabí. Manta. Manabí. Ecuador. inesveramolina@hotmail.com orcid.org/0000-0001-6933-455X
\end{abstract}

Nicole Salazar Menéndez. Graduada de la Universidad Laica Eloy Alfaro de Manabi. Manta. Manabi..Ecuador.nicole.sm.19@hotmail.com

Adriana Romero Zambrano. Graduada de la Universidad Laica Eloy Alfaro de Manabí. Manta. Manabi.Ecuador.aeromeroz@hotmail.com

Fecha de recibido: 2019-07-25

Fecha de aceptado para publicación: 2019-09-12

Fecha de publicación: 2019-09-30

\section{Resumen}

La adolescencia es una etapa de transición caracterizada por la búsqueda de identidad que produce un estado de vulnerabilidad para el adolescente, que necesita herramientas para insertarse en la sociedad acorde a su cultura. Es en la infancia, con sus relaciones parentales, donde se construyen los recursos que el adolescente usará. Desde el psicoanálisis, se puede entender que la función paterna es la que instaura el orden y, la ley en el sujeto. El objetivo de este estudio es analizar los efectos en la subjetividad de los adolescentes a causa de esta declinación. El estudio se realizó desde un formato cualitativo, fenológico. Se efectuó una revisión bibliográfica, aplicando como herramienta la entrevista a expertos, informantes claves y adolescentes de Unidades Educativas de Manta. Como resultado, en esta investigación se evidenció la repercusión de la declinación paterna dentro del sufrimiento de los adolescentes, además la carencia de interés de los padres en el área educativa, donde se espera que sea la institución, quien instaure este sentido de orden y ley, dejando de lado la propia participación paterna dentro de este ámbito. Finalmente se recomienda abordar estas problemáticas focalizando en los indicadores de habilidades sociales, académicas y valores.

Palabras clave: Declinación paterna; función paterna; habilidades sociales; subjetividad.

\begin{abstract}
Adolescence is a transition stage characterized by the search for identity that produces a state of vulnerability for the adolescent, who needs tools to enter society according to their culture. It is in childhood, with their parental relationships, where the resources that the adolescent will use are built. From psychoanalysis, it can be understood that the paternal function is what establishes order and, the law in the subject. The objective of this study is to analyze the effects on the subjectivity of adolescents because of this decline. The study was conducted from a qualitative, phenological format. A literature review was carried out, applying as an interview the experts, key informants and adolescents of Manta
\end{abstract}


Educational Units. As a result, this investigation showed the impact of the paternal decline in the suffering of adolescents, as well as the lack of interest of parents in the educational area where the institution is expected to establish this sense of order and law, leaving aside the own parental participation in this area. Finally, it is recommended to address these problems focusing on the indicators of social, academic and values skills.

Keywords: Parental decline; paternal function; social skills; subjectivity.

\section{Introducción}

Para hablar de la función paterna y subjetividad de los adolescentes es necesario hablar de la familia debido a que el cambio de su estructura mueve estas posiciones, explicando la función paterna, la adolescencia en la actualidad y cómo es visto el padre con todas estas variaciones en nuestro tiempo.

La adolescencia es una etapa transitoria del desarrollo vital del sujeto, Güemes-Hidalgo, Ceñal, Hidalgo (2017) sale de la niñez y busca su identidad como joven para ser adulto. Esta etapa de transición deja al sujeto en un estado de vulnerabilidad frente a los diversos cambios en todos los aspectos de su vida, encontrándose frente a una cantidad de nuevas circunstancias que harán que requiera de herramientas para poder insertarse en la sociedad y su cultura.

Esto se lograría desde su formación inicial dada por estas figuras paternales que establecen las primeras pautas de estructuración vital, es la familia el eje que mueve y estructura a la persona, sin embargo, en nuestro tiempo se han dado grandes cambios a nivel cultural e ideológico que han influido en el concepto tradicional de familia.

La familia como institución ha pasado por grandes cambios desde el modelo familiar, González (2013) su estructura y roles, eventos como: la liberación femenina y su inserción a los medios laborales, ha logrado que la mujer se vuelva independiente económicamente. Así lo manifiesta María Asunción González en sus estudios del 2005 en la crisis de la función paterna, menciona:

La progresiva incorporación de las mujeres al trabajo asalariado, el descubrimiento de los anticonceptivos -que permitió una mayor libertad femenina en el control de la propia vida-, y los cambios culturales que han acompañado tales transformaciones dibujan una familia en la actualidad muy lejana a su antecesora, y los roles desempeñados (p. 3).

La UNICEF (2002) menciona en Adolescencia. Una etapa fundamental que “... los jóvenes establecen su independencia emocional y psicológica, aprenden a entender y vivir su sexualidad y a considerar su papel en la sociedad del futuro, que es un proceso gradual, emocional y, a veces, perturbador" (pág. 4). Por lo que este periodo de vida resulta especialmente vulnerable en lo que respecta, al contacto que el adolescente establece con el Otro, entendido como ente social.

Los jóvenes adolescentes empiezan a enfrentarse con la ley social, lo que les obliga a inmiscuirse en la misma; es aquí donde surge el sentido de identidad psíquica que servirá de 
presentación para el joven en el mundo social expresado en "La adolescencia. Una época de oportunidades" de la UNICEF (2011).

Nasio (2010) investigó a 6 millones de jóvenes franceses, entre 11 y 18 años, clasificando el sufrimiento de los jóvenes en:

- moderado

- intenso

- extremo

En tanto el sufrimiento moderado, traducido como neurosis de crecimiento, se caracteriza por síntomas relacionados con la angustia, la tristeza y rebeldía, del estudio antes mencionado, se evidenció que 5 millones de jóvenes se identificaban con esta forma de vivenciar la adolescencia con problemas con poca gravedad.

Más adelante, con la característica de "intenso" se encuentran jóvenes que constantemente se deprimen, tienden a aislarse, realizan intentos de suicidio o se suicidan, hacen uso de sustancias psicotrópicas adictivas, pueden acceder a conductas alimentarias como la bulimia y la anorexia, realizan actos de vandalismo y pueden llegar a violentar a los demás o contra sí mismo. Un millón de adolescentes sienten y experimentan la adolescencia en relación con comportamientos peligrosos (Nasio, 2010).

Por último, el sufrimiento extremo alude al vivenciar de la adolescencia, a sujetos víctimas de perturbaciones mentales, como la depresión, síntomas del Trastorno obsesivo compulsivo, fobias, desórdenes alimentarios crónicos, perversiones sexuales, y síntomas relacionados con, o directamente con la presentación de la esquizofrenia, estas afecciones llegan a repercutir en la vida adulta de estos jóvenes de forma permanente.

Estas categorías señalarían de manera particular los tipos de vivencias afectivas que experimentan los jóvenes formados en las sociedades, además, explican la manifestación de síntomas y patologías, que en muchos casos se presentan por la vulnerabilidad latente en esta etapa.

Desde el psicoanálisis, Sigmund Freud explica que es el padre quien ejerce la función de inserción de las normas culturales, de la imposición de la ley y de "comprender de manera implícita la prohibición" (Sigmund, 1905).

Entonces si retomamos los cambios ideológicos que se han venido presentando a lo largo de la historia, nuestra forma de ser y de presentarse, ha ido transformando su perspectiva, llegando incluso a modificar estructuras simbólicas de la cultura, como las mismas funciones paternales. Y como resultado de estos cambios, se puede mencionar que, en la construcción de la subjetividad normativa actual, existen cambios fundamentales, a lo que se conocía hace años atrás y que fundaba generaciones de sujetos con características semejantes, como la de los antecesores de los mismos adolescentes: sus padres y sus abuelos.

Esta situación de vulnerabilidad dada por las características existenciales del periodo de vida que atraviesa el adolescente expone; más que todo en nuestra época; como se ha desplazado la importancia de los cambios avenidos en la sociedad (García, 2013) expresa: 
En la adolescencia la declinación del imago del padre y de los ideales a ella vinculados podría determinar un aumento en la mencionada vacilación creada por la pérdida de los referentes de la infancia, en la medida en que estos no son sustituidos por sistemas de ideas a partir del padre de la familia (o contra este) o de ideologías de fuerte impregnación cultural que ordenen todo el armado subjetivo (p.113).

La pregunta ¿qué es un Padre? Pese a ser un término usado de forma universal, el término padre ha ido cambiando y modificando su significado en base al tiempo histórico en que ha sido empleado, Philippe Julien en su libro El manto de Noe (1993) describe la evolución que ha tenido este nombre, en cuanto a su significado a lo largo de la historia desde un contexto sociocultural.

Tradicionalmente se ha ubicado al padre como figura de autoridad, de respeto, el que impone la ley, el que sabe o supuestamente sabe, el que protege, el que provee, el que brinda seguridad por su mayor fortaleza.

Esta es la contextualización que a partir del siglo XX se estableció para describir la paternidad, refiriéndose así a este padre cariñoso que mantiene un vínculo cercano y afectivo, que es partícipe no solo de su educación moral, sino que es responsable de cada aspecto de la vida de sus hijos. El padre ya no es aquel de quien la madre habla, sino que es él que habla a sus hijos. El lugar que ocupa actualmente el padre ha cambiado, ubicándolo más del lado de los afectos que de la autoridad Mitjáns (2006).

Para (Freud, 1905) el padre, proveedor, viril y castrador, que con su actuar viene a separar, a poner un límite en la relación que tienen mamá - hijo, por lo que Freud en su texto (yo, el súper yo y el ideal del yo) nombra como "el complejo de Edipo" a esta dinámica:

... del padre, el varoncito se apodera por identificación. Ambos vínculos marchan un tiempo uno junto al otro, hasta que, por el refuerzo de los deseos sexuales hacia la madre, y por la percepción de que el padre es un obstáculo para estos deseos, nace el complejo de Edipo (p. 33).

Siendo este quien realiza el corte simbólico es quien asume dentro de la dinámica un primer significante de orden y ley, ubicándose en una posición de autoridad para instituir al niño que no puede poseer a la madre y estableciendo cuál es su lugar dentro del ámbito familiar, esta ley que humaniza debería aportar al niño mecanismos que le permitan insertarse de acuerdo a las reglas en la sociedad, mostrándole que corresponde al "deber hacer".

Jacques Lacan estudia los conceptos de Saussure (1916) que hacen un señalamiento y distinción entre lengua y habla, lo abstracto del lenguaje evocaría en la facultad de los hombres para construir el lenguaje a través de la lengua y el habla sería el elemento intangible de la misma, que permite instaurarse en dependencia del contexto social. La lengua está ligada a lo abstracto del lenguaje, y el habla a lo material (Lacan, 2011).

El nombre del padre opera, como lo indica (Lacan, 2011) en sus seminarios, como la inscripción en el individuo de la ley humana que permite el acceso a la cultura, que es también el 
dominio del lenguaje, la palabra y su inserción a la misma. Para Murillo (2011) es importante el uso de la palabra, para nominar, para inscribir en el sujeto el constructo de significante.

Para ello se planteó en esta investigación la pregunta: ¿qué efectos produce la declinación de la función paterna en la subjetividad de los adolescentes de la ciudad de Manta? Por lo tanto, su objetivo es: analizar los efectos en la subjetividad de los adolescentes a causa de esta declinación.

\section{Metodología}

El tipo de investigación que se realizó es cualitativo, con un diseño fenomenológico empírico puesto que este diseño, explora, describe y comprende lo que los sujetos tienen en común de acuerdo con sus experiencias, relacionadas con un determinado fenómeno Hernández, Fernández \& Baptista (2014).

El método utilizado fue el análisis y síntesis. Las técnicas utilizadas fueron: revisión bibliográfica, entrevista semi dirigida a 8 informantes claves, es decir a los jefes del departamento estudiantil de las unidades educativas fiscales de manta; entrevista cerrada a 4 expertos y a 15 estudiantes de las unidades educativas fiscales de manta. Esta es una investigación de análisis fenomenológico sobre la función paterna y subjetividad en los adolescentes para obtener la información pertinente relativa a la temática de estudio.

\section{Resultados y Discusión}

Los expertos en las entrevistas indicaron que en la adolescencia las figuras parentales establecen alianzas que como parte de la subjetividad del adolescente; el grupo influye en cuanto la identidad, sentido de pertenencia social, ideología o metas. En este periodo se experimenta la sexualidad, la implicación del cuerpo, las relaciones amorosas y el consumo y abuso de sustancias de alcohol, drogas, etc.

Los informantes claves señalaron que, debido a la ausencia de los padres en las unidades educativas, como en el hogar, se da paso a una "función paterna ausente" mencionan que el "gestor de la autoridad" en el hogar no se manifiesta como se lo espera, generando que los adolescentes respondan agresivos, violentos, llegando a generar abusos como la violencia entre pares y el abuso sexual, daños psicológicos como el bullying, entre otros. Eso sí, se expresa que hay un grupo determinado de padres de familia que corresponden a los llamados institucionales y suelen estar pendientes de sus hijos, estos chicos están más adaptados a las exigencias en el colegio, correspondiendo a una "función paterna estable" donde los límites se experimentan de acuerdo a lo esperado.

\section{En la entrevista a adolescentes:}

Respecto al ámbito familiar, las funciones se mantienen desde distintas representaciones ya sea por otra pareja de parte de la madre o desde los abuelos.

En la variable de relación con los padres se evidencia tener poca o ninguna relación con el padre biológico, sin embargo, tienen una buena relación con la figura que representa la función paterna. De forma general se mantiene una buena relación con la madre o con quien ha ejercido la función materna. 
En la importancia a la figura paterna se señala que, dependiendo del vínculo establecido por los padres se evidencia la importancia de ellos en la adolescencia, que pese a las disconformidades consideran de suma importancia este puesto y lo valoran, siendo representada no necesariamente por los padres biológicos.

La expectativa sobre los padres, la mayoría de los jóvenes expresan tener alguna expectativa por parte de la función que ejercen ambos padres, evidenciando el deseo de que se sostenga el concepto de familia tradicional con las manifestaciones de actos y afectos que reconoce una familia normal.

Respecto al ideal familiar, es importante recalcar que se ha expresado la necesidad de manifestación del lazo familia, más allá del hecho de vivir bajo el mismo techo.

Entonces debido a estos indicadores propuestos se pudo evidenciar en el discurso de los adolescentes, recogido en las entrevistas con ellos, que:

Cuando en su dinámica familiar encontramos una función paterna estable, donde existe y está presente la figura que representa la función se encuentran adolescentes más ajustados a la norma, conscientes de la necesidad de los límites impuestos, tienen menos problemas para adaptarse a las exigencias planteadas por los medios de relación más cercanos, como lo son las unidades educativas y sus problemáticas tienden a ser resueltas con mayor efectividad, por lo que el tipo de sufrimiento que expresan y sienten respecto a su vida cotidiana, es moderado, ya que es funcional dentro de las condiciones en las que se encuentra, como lo indica Nassio (2016).

Un adolescente con una función paterna estable, que tiene un sufrimiento intenso puede llegar a cuestionarse sobre la "ley", pero el núcleo familiar de apoyo se encuentra sosteniendo estas crisis que podrían atacar la vida cotidiana de los adolescentes Organización Mundial de la Salud (2019).

Sin embargo, en un caso donde la función paterna se encuentra y se sostiene estable pero que el adolescente se encuentra en una dinámica de sufrimiento extremo, se podrían considerar características psicopatológicas de base que predisponen esta vulnerabilidad ante cualquier crisis que el sujeto pueda experimentar en este periodo, pero vale recalcar, que casos como aquellos son inusuales y no se hallaron en la investigación evidencia de los mismos, por lo que, si se encontrase caso alguno también sería pertinente observar de manera puntual, la forma en la que se relacionan los padres con el adolescente.

Tomando el segundo indicador de la tipología de función paterna, cuando esta se encuentra declinada y hay en el adolescente una presentación de sufrimiento moderado, sus experiencias tienden a volverse problemáticas, ya que por la misma característica de las "crisis" en este periodo, que falte o se encuentre de manera ambivalente la figura de ley, genera en los adolescentes mayor dificultad para controlar y entender sus emociones y por ende para responder ante las exigencias del medio, por lo que estos chicos están propensos a ser guiados o influidos por otros referentes encontrados en sus pares.

Otra manifestación de la función paterna declinada, alude a cuando el sujeto joven se encuentra en un estado de sufrimiento intenso, se les suele denominar en las instituciones como "chicos problemas" puesto que la presencia ambivalente de la figura paterna y una sensación de sufrimiento intenso, ya que en el discurso de los jóvenes se encuentra la necesidad de que exista 
esta ley y debido a su falta, ellos tienden a regirse ante sus ideales consecuentes, pero que suelen dirigirse a situaciones y problemáticas que son desfavorables para su vida cotidiana. Pueden llegar al consumo de sustancias, vínculos con pandillas, actos delictivos, intentos de suicidio o el mismo suicidio y todo esto deviene de la necesidad de tener una ley o referente de ley que al no estar presente, fue asumida por sí mismo.

Siguiendo la lógica de la tipología de función paterna declinada y un sufrimiento extremo se puede inferir que estos jóvenes tendrán problemas particulares, ya que las consecuencias que dejan al adolescente en una dinámica poco funcional que repercute en sus vivencias cotidianas, tomando en cuenta la predisposición de los mismos a presentarse un cuadro psicopatológico que afectaría de forma contundente en su vida y si el referente de la ley y sostén no se encuentra presente, sería uno de los factores que predispongan al sufrimiento del sujeto, llevándolo a la búsqueda de satisfacer esas necesidades, pudiendo caer en el consumo problemático de sustancias psicotrópicas fuertes que conllevarían a casos de adicción a temprana edad. Jaramillo (2007).

Más adelante también se refiere sobre una función paterna ausente, o también inexistente, puesto que no hay figura que represente la función, las razones por las que no está, pueden darse o por muerte o abandono del padre a la familia. Hay que mencionar que al dejar este lugar vacío dentro de la dinámica de cualquier familia, es perjudicial y disfuncional para los hijos, puesto que deja secuelas en su formación de forma permanente, es por esto, que en un sufrimiento moderado de la adolescencia y que el rector organizador de la ley no se encuentre, genera dificultades para seguir las normativas y serán problemáticos en sus relaciones con el entorno, sin embargo otras fuentes de apoyo, $\mathrm{u}$ otras instituciones que logren simbolizar la ley, pueden ubicar al adolescente dentro de la normativa del contexto en el que se desenvuelva Lacan (2011).

Sin embargo, los adolescentes con sufrimiento intenso sin la figura de la función genera chicos que por la necesidad de encontrar algo que los organice se involucrarán en diversas dinámicas disfuncionales que en consecuencia dejarán a los adolescentes sin ideales a los cuales referirse y sus proyectos y planificaciones de vida se verán empobrecidos, siendo vulnerables en mayor medida a generar familias a temprana edad y abandonarlas, siguiendo el mismo patrón aprendido, delinquiendo, consumiendo sustancias, instalándose como los dueños de su ley, una ley pervertida e insuficiente que en algunos casos podría generar patologías por esta función ejecutada por el sujeto, sin orden.

Por último, en el caso particular donde el sufrimiento es extremo y la ausencia del padre, tanto en función como en figura, vemos que genera a nivel de estructura disfuncionalidad, dando apertura a la presentación y desencadenamiento de psicopatologías, como lo es la psicosis, que repercuten en el sujeto para toda su vida, ya que al nos inscribirse de ninguna manera la "ley", estos chicos tratan de encontrar algo que organice y ordene su sentido de la vida Nassio (2016) en su texto sobre la adolescencia nos indica los tres tipos de adolescentes haciendo referencia en este punto al tercer tipo, es decir, al adolescente que sufre ya alguna psicopatología por lo tanto requiere de tratamiento psicológico.

Actualmente la familia tradicional ha cambiado completamente, componiéndose de diversas formas separando el cuerpo de la función, sin embargo, la perspectiva de los adolescentes sobre la función paterna indica que es crucial en su vida ya que se hace referencia a la importancia de la misma pero sobre todo desde los distintos cuerpos en que esta pueda tomar lugar, poniendo en 
evidencia el malestar ocasionado por la declinación de esta función desde sus progenitores quienes serían los encargados de llevar a cabo esta función y no lo hacen, o lo hacen de manera insuficiente creando un malestar que repercute a nivel familiar, no solo al nivel del padre sino también el lugar de la madre y del propio adolescente alterando este pequeño sistema. Pese a esto sostenemos la importancia que se le ha dado a quienes han asumido esta función declinada, que al hacerlo como se supone que está dentro del ideal para el adolescente, presenta una particular conformidad dentro de su descontento natural y su aislamiento de la familia, el vínculo que se establece es la base de la relación que permitiría un curso adecuado de la dinámica familiar.

\section{Conclusiones}

Como resultado en nuestra investigación, fue evidente la repercusión de la declinación paterna tomando un papel significativo dentro del sufrimiento de los adolescentes actuales. En la entrevista a los informantes se corrobora la carencia de interés en los padres dentro del área educativa donde se espera que sea la institución, quien instaure este sentido de orden y ley, dejando de lado la propia participación paterna dentro de este ámbito.

En esta etapa cambiante que permite el moldeamiento de la identidad concierne en gran medida a las relaciones que tendrá en este periodo y tomando en cuenta las diferentes tendencias culturales que pueden influir en el adolescente haciendo que se presenten pocos mecanismos de control y una carencia en la función paterna ejecutada por los padres que repercute en la forma en que el adolescente estructura su subjetividad.

También podemos concluir que aunque se encuentra una función paterna estable con un sufrimiento moderado dentro del medio estudiado también se encuentra una incidencia de una función paterna declinada con un sufrimiento moderado ya que el padre está presente en tanto forma, pero se encuentra ausente en el hogar en tanto a su función, dando paso a la demanda de parte de sus hijos; y una función paterna declinada con sufrimiento intenso que se lo evidencia en una reacción disfuncional ante las problemáticas que puedan surgirle.

Como podemos ver en consecuencia la subjetividad de los adolescentes donde hay sufrimiento moderado e inclusive intenso su subjetividad se ve afectada en el sentido que no puede pasar adecuadamente su proceso de adolescencia, repercutiendo su comportamiento en lo afectivo, en su sexualidad, en lo social y en lo académico

De las relaciones establecidas en tanto funciones existe una declinación de la función paterna que representa sufrimiento considerable para el adolescente siendo un factor de riesgo para la construcción de una subjetividad que le permita desarrollarse dentro de la normativa social con mayor tendencia a la vulnerabilidad en sus afectos.

Se elaboró la propuesta de una ficha de observación para los estudiantes de las unidades educativas con la finalidad de que sirva como modelo para un mejor estudio de los adolescentes en las habilidades académicas, sociales y sus valores, permitiendo tener acceso a una recopilación de datos más completa y especifica; este modelo podrá ser usado para futuras investigaciones dentro de los proyectos afines. 


\section{Otras consideraciones}

Cabe recalcar que este trabajo investigativo está insertado en el Grupo de Investigación en Psicoanálisis de la Facultad de Psicología de la Universidad Laica Eloy Alfaro de Manabí, desde el año 2017, en su línea de investigación sobre las psicopatologías.

\section{Referencias Bibliográficas.}

Casas, J.; Ceñal, M.; (2005) Desarrollo del adolescente. Aspectos físicos, psicológicos y sociales. Pediatr Integral 2005; IX (1):20-24. Unidad de Medicina del Adolescente.

Servicio de Pediatría. Hospital de Móstoles, Madrid. Recuperado de:

http://www.sld.cu/galerias/pdf/sitios/puericultura/desarrollo_adolescente(2).pdf

Freud, S (1905) Tres ensayos sobre la sexualidad. Obras completas. Recuperado de:

http://www.cieg.unam.mx/lecturas_formacion/identidad_imaginaria/Tema_III/

Sigm ound_Freud_Tres_Ensayos_sobre_la_sexualidad.pdf

García, J. (2013) Los adolescentes, la declinación del patriarcado y las nuevas estructuras familiares. Revista de Uruguaya de Psicoanálisis. Uruguay.

González, A. (2013) Los conceptos de patriarcado y androcentrismo en el estudio sociológico y antropológico de las sociedades de mayoría musulmana. Collège de France/EHESS. Laboratoire d'Anthropologie Sociale. Papers 2013, 98/3.

González, M. (2005) La crisis de la función paterna, el nuevo lugar/deseo de las mujeres y el ejercicio de la parentalidad. Revista de comunicación interactiva.

Güemes-Hidalgo, M.; Ceñal, M.; Hidalgo, M. (2017) Pubertad y adolescencia. ADOLESCERE. Revista de Formación Continuada de la Sociedad Española de Medicina de la Adolescencia. Volumen V. Enero-febrero 2017. No 1.

Hernández, R., Fernández, C., \& Baptista, M. (2014). Metodología de la investigación McGrawHill. México DF.

Jaramillo, L. (2007) Concepciones de infancia. Revista del instituto de estudios superiores en educación. Universidad del Norte. Zona Próxima, núm. 8, 2007, pp. 108-123

Lacan, J. (2011) Seminario 5. Las formaciones del inconsciente. Recuperado de: http://www.bibliopsi.org/docs/lacan/Seminario-5-Las-Formaciones-DelInconsciente-Paidos-BN.pdf

Mitjáns, (2006) Trabajo infantile y subjetividad: una perspectiva necesaria. Estudios de psicología. Universidad Federal de Río Grande del Norte. Natal, Brasil. ISSN 1413 - 294X.

Murillo, M. (2011) La hipótesis de los tres registros - simbólico, imaginario, real - en la enseñanza de J. Lacan. Anuario de Investigaciones, vol. XVIII, 2011, pp. 123132 
Nasio, J. (2010) ¿Cómo actuar con un adolescente dificil? Consejos para padres y profesionales. 1era edición. Buenos Aires; Paidos, 2010.

Organización Mundial de la Salud. (2019) Desarrollo en la adolescencia. Artículo de la web. Recuperado de:

https://www.who.int/maternal_child_adolescent/topics/adolescence/dev/es/

UNICEF (2002) Adolescencia. Una etapa fundamental. Nueva York. Fondo de las Naciones Unidas para la Infancia .Recuperado de: https://www.unicef.org/ecuador/pub_adolescence_sp.pdf

UNICEF (2011) La adolescencia. Una época de oportunidades. (C) Fondo de las Naciones Unidas para la Infancia. Febrero de 2011. ISBN: 978-92-806-4557-6. Recuperado de: https://www.unicef.org/bolivia/UNICEF_-

_Estado_Mundial_de_la_Infancia_2011_La_adolescencia_una_epoca_de_opor tunidades.pdf 